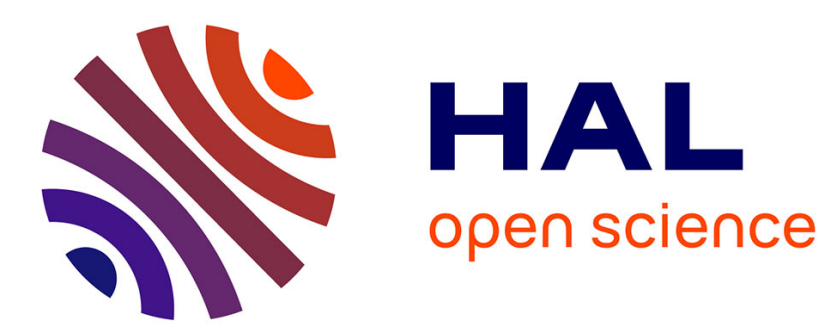

\title{
Renormalisation groups with periodic and aperiodic orbits
}

\author{
Bernard Derrida, J.-P. Eckmann, A. Herzan
}

\section{To cite this version:}

Bernard Derrida, J.-P. Eckmann, A. Herzan. Renormalisation groups with periodic and aperiodic orbits. Journal of Physics A: Mathematical and Theoretical, 1983, 10.1088/0305-4470/16/5/009 . hal-03285945

\section{HAL Id: hal-03285945 \\ https://hal.science/hal-03285945}

Submitted on 21 Jul 2021

HAL is a multi-disciplinary open access archive for the deposit and dissemination of scientific research documents, whether they are published or not. The documents may come from teaching and research institutions in France or abroad, or from public or private research centers.
L'archive ouverte pluridisciplinaire HAL, est destinée au dépôt et à la diffusion de documents scientifiques de niveau recherche, publiés ou non, émanant des établissements d'enseignement et de recherche français ou étrangers, des laboratoires publics ou privés. 


\title{
Renormalisation groups with periodic and aperiodic orbits $\dagger$
}

\author{
B Derridał, J-P Eckmann§ and A Erzan\$ \\ ‡ Service de Physique Théorique, CEN Saclay, 91191 Gif-sur-Yvette, France \\ \$ Département de Physique Théorique, Université de Genève, 1211 Genève 4, Switzerland
}

Received 11 August 1982

\begin{abstract}
Motivated by recent results for some frustrated hierarchical models, we analyse the consequences of renormalisation group transformations with periodic and aperiodic orbits. We find that the free energy has in general an infinite number of singularities. This occurs already when the transformation has a stable periodic orbit. We present an idealised example, and a new class of hierarchical Potts models to which the theory applies.
\end{abstract}

\section{Introduction}

One of the achievements of renormalisation group theory has been the reduction of problems of phase transitions and critical phenomena to the study of maps in a space of Hamiltonians (Wilson and Kogut 1974, Kadanoff 1976). These maps are introduced by looking at the effective Hamiltonian which is obtained from a given Hamiltonian when some degrees of freedom are eliminated in the formation of the partition function. This approach is so successful because it allows us to relate phase diagrams and critical behaviour to properties of the map: an attractive fixed point represents a thermodynamic phase, a hyperbolic fixed point represents a phase transition, the critical exponents are connected by simple relations to the linearised map around the hyperbolic fixed points.

It is most often impossible to find tractable expressions for the renormalisation map. To apply nevertheless the ideas of the renormalisation group theory, one usually replaces the map defined in the whole space of Hamiltonians by an approximate map in a subspace of finite dimension. This approximate map is often adequate to describe the phase diagram completely and may lead to rather accurate estimates of the critical exponents. It has been known for a long time that some of these approximate renormalisation groups have exact realisations on hierarchical lattices. For some more recent developments relevant to our context see Berker and Ostlund (1979), Kaufman and Griffiths (1981) and McKay et al (1982). The hierarchical lattices of interest here are generated by recursively replacing each bond connecting two sites by a network of bonds according to a uniform prescription. The renormalisation procedure then consists of decimating the degrees of freedom residing at the vertices of this network, to give rise to a renormalised bond.

\footnotetext{
$\div$ Partially supported by the Swiss National Science Foundation.

$\|$ On leave of absence from the Division of Theoretical and Applied Mechanics, Istanbul Technical University.
} 
Maps of a space to itself have been studied in many contexts, and it is by now well known (May 1976, Collet and Eckmann 1980) that even very simple maps may have behaviour which is more complex than the one described above: one may find periodic orbits, strange attractors, absolutely continuous invariant measures, .... An important question is to know if the renormalisation group (RG) map may at all present such complicated behaviour and what would be the physical consequences for the phase diagram and the critical behaviour. This question would of course be only an academic one if the RG maps never had other periodic orbits than fixed points. It has, however, been recently discovered (McKay et al 1982) that, at least for some frustrated hierarchical models, the RG map has periodic orbits or chaotic behaviour. It is reasonable to hope that other physical systems will be shown to have similar RG maps. Therefore we found it interesting to try to understand the consequences of periods and of chaotic behaviours of the RG map on the phase diagram and on the critical properties.

The purpose of this paper is to study the analytic properties of the free energy when the RG map has periodic orbits or chaotic behaviour. To simplify the discussion, we shall restrict ourselves to the case where the dimension of the space of Hamiltonians is one, i.e. the only parameter in the problem is the 'temperature' $t$. We suppose that we know the RG map $T(t)$ which corresponds to a change of scale $a$ (the constant $a$ is always larger than one). At each change of scale the temperature $t$ is changed into a renormalised temperature $T(t)$ and one usually obtains an analytic contribution $g(t)$ to the free energy $f(t)$. The free energy is then given by (Niemeijer and van Leeuwen 1976)

$$
f(t)=\sum_{n=0}^{\infty} a^{-n} g\left[T^{n}(t)\right],
$$

where $T^{n}(t)$ means the $n$th iterate of $t$ :

$$
T^{n}(t)=T\left[T^{n-1}(t)\right], \quad T^{0}(t)=t .
$$

It is well known in the theory of critical phenomena that, even if $g$ and $T$ are analytic around a repulsive fixed point $t_{0}$ of the map, the sum (1) acquires a singular part in the free energy at this fixed point $t_{0}$. The nature of this singularity is usually easy to understand by rewriting (1) as

$$
f(t)=g(t)+a^{-1} f[T(t)]
$$

and by looking for the power law singularity of $f(t)$ at the fixed point $t_{0}$ which is compatible with (3). In the present paper, we want to generalise this idea to cases where the map $T(t)$ has periodic orbits or chaotic behaviour.

When the map $T$ has an attractive period, the situation does not seem, at first sight, very different from the case of an attractive fixed point. There are, however, two main reasons which make the phase diagram much richer. First, from topological reasoning, like the Sarkovskii (1964) theorem, one knows that the existence of a periodic point for a continuous one-dimensional map implies that the map has a lot of other periodic points, most of them being in general unstable. At each temperature belonging to one of these unstable periods, the free energy is singular. Second, by looking at (3), it is clear that if $f(t)$ is singular at a point $t_{1}$, then it is also singular at all the pre-images of $t_{1}$. This means that an unstable period brings all its pre-images 
in the set of critical temperatures. When the map is not monotonic there will in general be many such pre-images. A consequence is that in a situation like the one studied by McKay et al (1982), even when the RG map has only an attractive period 2 , the free energy has an infinite number of singularities because the unstable fixed point has an infinite number of pre-images. On the other hand, when the map has no attractive periods at all (such maps exist), then under some additional technical conditions the pre-images of the unstable periodic points are dense in some regions of temperature. In these regions the free energy will exhibit singularities at a dense set of temperatures.

This paper is organised as follows. In $\S 2$, we recall a few notions which are useful in the study of maps. The experts are encouraged to skip it. In $\S 3$, we study the analyticity properties of the function $f(t)$ defined by equation (1). We identify the singularities and the behaviour of $f(t)$ in the neighbourhood of these singularities. For a very simple choice of the map $T(t)$ and the function $g(t)$, we present the numerical calculations of the function $f(t)$ in several situations: the map $T$ has simple attractive periods, or no attractive period at all. We shall see that even for our simple choice of the $T(t)$ and $g(t)$, the sum (1) gives for $f(t)$ a very complicated analytic structure. In $\S 4$, we give some general results about the analytic properties of the function $f(t)$ for a general choice of $g(t)$ and $T(t)$. Finally in $\$ 5$ we exhibit a simple hierarchical model similar to those studied in McKay et al (1982) and Śvrakić et al (1982) and exhibiting the general features discussed before. We find accumulation of critical behaviour, although the singularities are weak and only high derivatives of $f$ diverge.

\section{Properties of one-dimensional maps}

The problem of maps of the interval has been studied by many authors in the recent past. We just want to recall here a few notions which are useful to understand the following sections.

\subsection{Fixed points, periods and pre-images}

$T$ is a real analytic map of the real axis to itself. A set of $p$ distinct points $t_{1}, t_{2}$, $t_{3}, \ldots, t_{p}$ forms a period (or a periodic orbit) of length $p$ if the map $T$ acts on these points like a circular permutation

$$
t_{i+1}=T\left(t_{i}\right), \quad 1 \leqslant i<p, \quad t_{1}=T\left(t_{p}\right) .
$$

A fixed point is a period of length 1 . The pre-images of a point $t_{0}$ are the set of points $t$ which are mapped onto $t_{0}$ by $T$ after a finite number of iterations.

A period $t_{1}, t_{2}, \ldots, t_{p}$ of length $p$ is called stable if under the action of $T$ all the points of an open neighbourhood of $t_{1}, t_{2}, \ldots, t_{p}$ converge to this cycle under the action of $T$. By linearising the map $T^{p}$ around any one of the points of the period, one finds that a period $p$ is stable if

$$
-1<\prod_{i=1}^{p} \frac{\mathrm{d} T}{\mathrm{~d} t}\left(t_{i}\right)<1
$$

The basin of attraction of an attractive period is the set of all the points which tend to this period. 
Conversely, a period is called unstable if

$$
\left|\prod_{i=1}^{p} \frac{\mathrm{d} T}{\mathrm{~d} t}\left(t_{i}\right)\right|>1 .
$$

Any point in a neighbourhood of an unstable period moves away under the action of $T$. It may, however, come close to this period after some more iterations of $T$.

\subsection{On the abundance of periodic points}

The assumption that the map $T$ is a continuous function of $t$ on an interval has very striking consequences. For example, if $T$ has a period 3 one can show (Šarkovskii 1964) that it necessarily has an infinity of other periods, and that the number of periods of length $\leqslant n$ must grow exponentially (in fact like $\left.[(1+\sqrt{5}) / 2]^{n}\right)$. Similar statements hold with another exponential growth constant whenever $T$ has at least a period which is not a power of 2 .

\section{Analyticity and singularities of the free energy}

We are now going to see how the properties of the map determine the analytic structure of the function $f$. In this section, we intend to give a more heuristic description of the results, some of which will be described in more detail in the next section.

\subsection{An unstable fixed point}

It is well known in the theory of critical phenomena that even if the function $g(t)$ and the map $T$ are analytic at an unstable fixed point $t_{0}$, the function $f(t)$ defined by (1) is in general singular at this unstable fixed point $t_{0}$ (see the review by Niemeijer and van Leeuwen (1976) and the references given there). The simplest way to compute the critical behaviour of $f(t)$ is to realise that if $f(t)$ has a singular part $f_{\text {sing }}(t)$ at the point $t_{0}$, then this singular part must verify

$$
f_{\text {sing }}(t)=a^{-1} f_{\text {sing }}[T(t)] .
$$

This relation follows directly from equation (3) and from the fact that $g(t)$ is regular at $t_{0}$. One should notice that equation (7) does not prove that $f_{\text {sing }}$ exists. This depends on the choice of the function $g(t)$ and on properties of $T$. Let us just say that the generic situation is that $f_{\text {sing }}$ exists and satisfies (7).

We next derive the behaviour of $f(t)$ for $t$ near $t_{0}$. By linearising $T(t)$ at the unstable fixed point $t_{0}$,

$$
T(t)-t_{0} \sim \lambda\left(t-t_{0}\right), \quad|\lambda|>1,
$$

one obtains from (7) that the singular part of $f$ has the following form near $t_{0}$ :

$$
f_{\text {sing }}(t) \sim\left|t-t_{0}\right|^{\psi} h\left(\log \left|t-t_{0}\right| / \log |\lambda|\right),
$$

where the critical exponent $\psi$ is given by

$$
\psi=(\log a) / \log |\lambda|
$$


and the function $h$ is a priori any periodic function (the function $h$ is also usually different for $t-t_{0}>0$ and $t-t_{0}<0$ ),

$$
h(z+1)=h(z) \text {. }
$$

The function $h$ gives an oscillatory modulation of the critical behaviour of $f$. However, in the theory of critical phenomena, $h(z)$ is usually replaced by a constant because in other models the change of scale is arbitrary (Niemeijer and van Leeuwen 1976). As we shall see in our numerical example (\$3.6) and in a hierarchical model ( $\$ 5)$, the change of scale is no longer arbitrary and $h(z)$ is no longer a constant. A rather general situation will be that the unstable fixed point $t_{0}$ is an accumulation point of other critical points, for example the pre-images of another unstable fixed point $t_{0}^{\prime}$. Therefore, the function $h$ contains the singularities due to these pre-images and describes how these secondary singularities accumulate at the point $t_{0}$.

We shall now discuss several of the possibilities.

\subsection{An unstable period}

Suppose that the map $T$ has an unstable period $\left\{t_{1}, t_{2}, \ldots, t_{p}\right\}$ of length $p>1$. Then each of these $p$ points is a fixed point of the map $T^{\nu}(t)$. Therefore, one can write equation (3) as

$$
f(t)=G(t)+a^{-p} f\left[T^{p}(t)\right]
$$

with

$$
G(t)=\sum_{q=0}^{p-1} a^{-q} g\left[T^{q}(t)\right]
$$

The problem has exactly the form discussed in $\S 3.1$. So if the function $f(t)$ has a singular part at a point $t_{i}$ of an unstable period, it will have the form

$$
f_{\text {sing }}(t) \sim\left|t-t_{i}\right|^{\psi} h_{i}\left(\log \left|t-t_{i}\right| / \log |\lambda|\right)
$$

where $\lambda$ is the slope of the map $T^{p}$ at the point $t_{i}$ :

$$
\lambda=\prod_{i=1}^{p} \frac{\mathrm{d} T}{\mathrm{~d} t}\left(t_{i}\right)
$$

the exponent $\psi$ is given by

$$
\psi=p(\log a) / \log |\lambda|
$$

and the function $h_{i}$ is again a periodic function of period 1 (which may again contain singularities)

$$
h_{i}(z+1)=h_{i}(z)
$$

One should notice that due to relation (3), all the points of the unstable period have the same critical exponent $\psi$ and the functions $h_{i}$ are related in the following way:

$$
h_{i}\left(\frac{\log \left|t-t_{i}\right|}{\log |\lambda|}\right)=a^{-1} \mid T^{\prime}\left(t_{i}\right)^{\psi} h_{i+1}\left(\frac{\log \left|t-t_{i}\right|+\log \left|T^{\prime}\left(t_{i}\right)\right|}{\log |\lambda|}\right) .
$$

For a period $p$, there are $p$ such relations (with $h_{p+1}=h_{1}$ ) which are compatible because $\lambda$ is given by equation (15). 


\subsection{Pre-images of an unstable period}

The equation (3) implies also that if $f$ has a singularity at a point $t_{0}$, it is also singular at all its pre-images $t_{-1}, t_{-2}, \ldots, t_{-n}$. Therefore all the pre-images of an unstable fixed point or an unstable period have the same critical exponent given by $(10)$ or (16), and their functions $h$ are related in the same way as in equation (18).

\subsection{An attractive period}

If the map $T$ has an attractive period and $T$ and $g$ are analytic, then the function $f$ is analytic in the basin of attraction of the period. This will be proved in $\S 4$. Even if the attractive period is short, one knows from the study of maps that the basin of attraction is very complicated: it is usually composed of an infinite number of open intervals. The boundaries of these intervals are the points of the unstable periods, their pre-images and their accumulation points. Therefore the function $f$ has in general an infinite number of singularities.

\subsection{No attractive period}

One knows that it is possible, and easy, to construct maps without any attractive period. In this case we shall show that, loosely speaking, $f$ is nowhere analytic. The condition for this will be that the pre-images of the periodic points are dense, which is true in vast classes of maps. There will be two subcases; in the first, the sum $\sum \partial_{t}^{k}\left\{g\left[T^{n}(t)\right]\right\} a^{-n}$ for some $k$ th derivative of $f$ diverges at a dense set of points. In the second case this sum diverges at more and more points as $k$ is increased, and only in the limit is a dense set reached.

It should be noted that all these statements are only true for generic $g$.

\subsection{A simple example}

To illustrate the behaviours explained above, we have chosen a very simple example which does not come from a hierarchical model, but which has the same qualitative features: the map $T$ is the quadratic map

$$
T(t)=\mu t(1-t), \quad 0 \leqslant \mu \leqslant 4,
$$

and the function $g$ is chosen to be

$$
g(t)=t \text {. }
$$

One knows that the quadratic map may have attractive periods or chaotic behaviour depending on the value of $\mu$. We have fixed the value of $a$ to be rather small,

$$
a=1.01,
$$

because we wanted to be able to see the singularities of $f$ in $f$ itself. Of course the choice (19), (20) and (21) is not supposed to represent a well defined physical situation, and the function $f(t)$ that we calculated numerically using equation (1) has no reason to present the usual convexity of a free energy. However, our choice was motivated by the fact that we wanted to show that even a very simple choice of $T(t)$ and $g(t)$ may give a very complicated analytic structure for the function $f$. The following cases are some representative examples. 
Case $A: \mu=3.236$. There is an attractive period 2 and two unstable fixed points at $t=0$ and $t=1-1 / \mu$. We have drawn in figures $1(a)$ and $1(b) f(t)$ as a function of $t$, and of $\log t$. We see the singularities occurring at the pre-images of the unstable fixed point $1-1 / \mu$. These singularities accumulate geometrically to the other fixed point $t=0$, and thus in a logarithmic scale they are equally spaced.
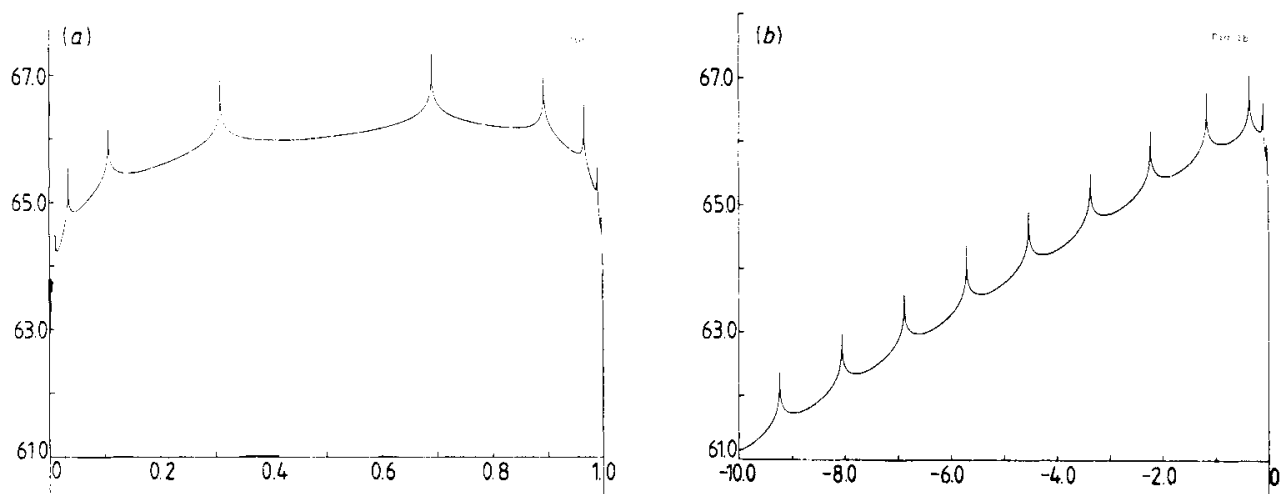

Figure 1. (a) The function $f(t)=\Sigma_{n=0}^{\infty} a^{-n} g\left[T^{n}(t)\right]$ with $g(t)=t, T(t)=\mu t(1-t)$ and $a=1.01$, for $\mu=3.236$ (stable period 2). (b) The same function plotted on a logarithmic scale. See $\S 3.6$, Case A.

Case $B: \mu=3.49$. There is an attractive period 4 , two unstable fixed points and an unstable period 2. In figure 2 we show $f(t)$ as a function of $t$; the peaks are the singularities located at the pre-images of one of the two unstable fixed points. The dips are other singularities located at the pre-images of the unstable period 2. One sees clearly that the dips accumulate to the peaks. Therefore the singular behaviour of the function $f$ near a peak is modulated (see equation (9)).

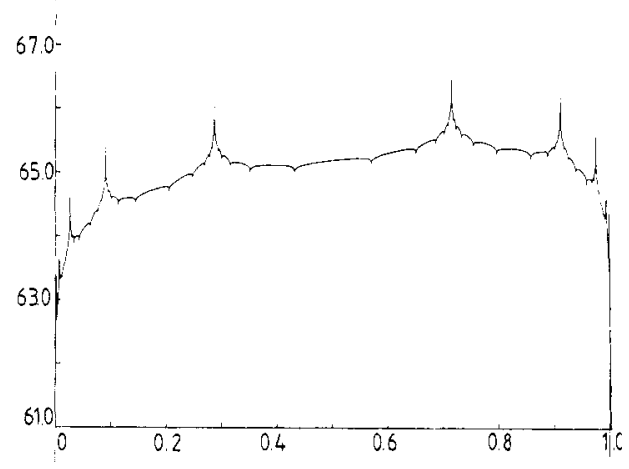

Figure 2. The function $f(t)$ for $\mu=3.49$, with stable period 4. See $\S 3.6$, Case B.

Case $C: \mu=3.8320$. There is an attractive period 3. One knows that there exist an infinite number of unstable periods. The function $f$ (see figure 3 ) has a lot of singularities corresponding to these unstable periods and also regions of analyticity corresponding to the basin of attraction of the period 3 . 


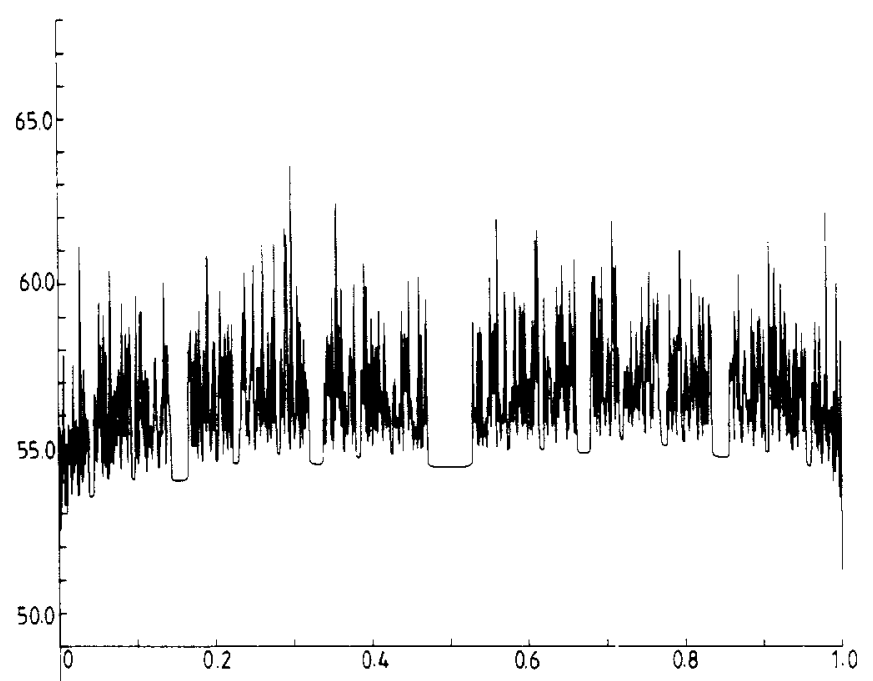

Figure 3. The function $f(t)$ for $\mu=3.832$, with stable period 3. See $\S 3.6$, Case C.

Case $D: \mu=4$. All the periods are unstable and there exists an absolutely continuous invariant measure. The function $f$ (see figure 4 ) seems to be nowhere analytic.

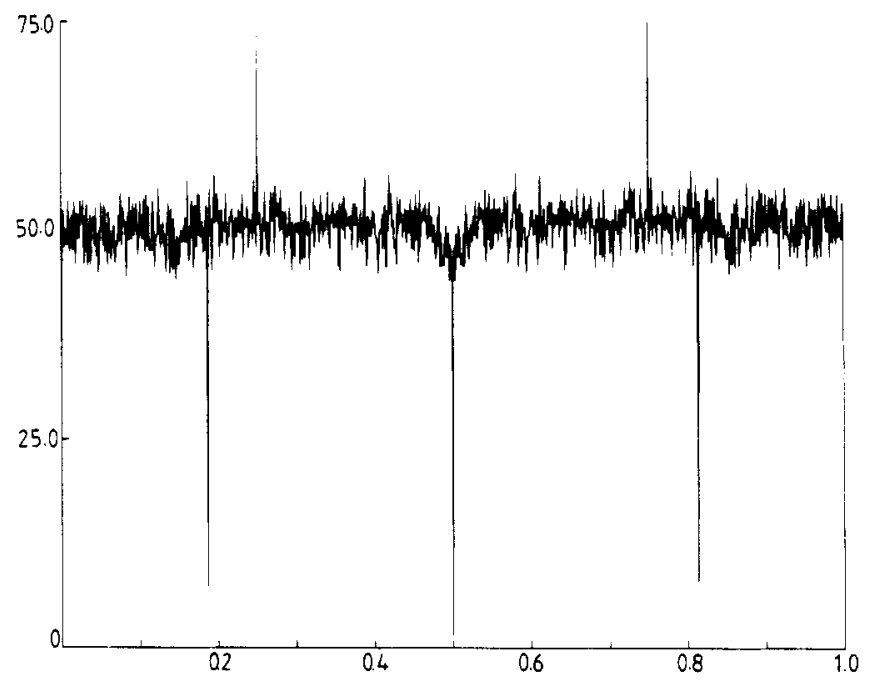

Figure 4. The function $f(t)$ for $\mu=4$, with no stable periods. See $\S 3.6$, Case D.

\subsection{A word of caution}

It cannot be sufficiently stressed that the exchange of sums and derivatives has disastrous effects in this problem. We hope that the following example will illustrate this. Suppose $T$ is given, and let $F$ be an analytic function. Define $g(t)=$ 
$F(t)-(1 / a) F[T(t)]$. Then it is easy to see that

$$
f(t)=\sum_{n=0}^{\infty} a^{-n} g\left[T^{n}(t)\right]=F(t),
$$

where this sum converges in general only on the interval which $T$ maps into itself.

As an example, take

$$
T(t)=4 t(1-t), \quad g(t)=t-4 a^{-1} t(1-t) .
$$

Then $f(t)=t$, but it is easy to see that

$$
\sum_{n=0}^{\infty} \frac{\mathrm{d}^{2}}{\mathrm{~d} t^{2}} a^{-n} g\left[T^{n}(t)\right]
$$

diverges at all points $t$ for which $T^{k}(t)=0$ for some $k$, if $a<4$.

Extending the above construction, one can show that by a small change of any given $g$ one obtains a new $\tilde{g}$ such that the function $\tilde{f}$ associated to it is analytic. We nevertheless believe that for a generic $g, f$ is not analytic at the pre-images of the periodic points.

\section{Some mathematical considerations}

As we have seen in the preceding sections, the object of study is the function

$$
f(t)=\sum_{n=0}^{\infty} a^{-n} g\left[T^{n}(t)\right],
$$

where $a>1$. In this section, we want to restrict the class of $T$ 's and $g$ 's in such a way that some precise statements are possible. It should be kept in mind that the study of (1) is a difficult subject and that the results presented here are of a preliminary and somewhat trivial nature. Our assumptions are as follows.

(1) Assumptions on $T$. The function $T$ maps the interval $[0,1]$ into itself, and is strictly increasing on $[0,1 / 2]$, and strictly decreasing on $[1 / 2,1] . T$ extends to an analytic function on a neighbourhood $\mathfrak{N}$ of $[0,1]$. (The choice of $1 / 2$ as a critical point is for convenience only.) The functions $T(t)=\mu t(1-t), \mu \in[0,4]$ satisfy all these assumptions, and so do, up to normalisation, the functions obtained in $\S 5$.

(2) Assumption on $g$. The function $g$ is analytic on the neighbourhood $\mathfrak{R}$.

Lemma 1. The sum (1) defining $f$ exists for all $t \in[0,1]$.

This is obvious, since $g$ is bounded. Note however, that, in general, the sum is not analytic in $t$, because the convergence is not uniform (in general).

Lemma 2. Assume $T$ has a stable fixed point, at $t_{0}$. Then $f$ is analytic in a neighbourhood of $t_{0}$, and its pre-images.

Proof. (NB All proofs are only sketched, but should be obvious from the sketch.) Since $t_{0}$ is a stable fixed point and $T$ is analytic, $T$ contracts a neighbourhood of $t_{0}$ into itself. Thus the family $\left\{g_{n}\right\}, g_{n}(t)=g\left[T^{n}(t)\right]$ is uniformly bounded on this neighbourhood and hence the sum is analytic. 
Lemma 3. Assume $T$ has an unstable fixed point at $t_{0}$. Then there is generically in $g$ a $k$ for which

$$
\sum_{n=0}^{\infty} a^{-n} \frac{\mathrm{d}^{k}}{\mathrm{~d} t^{k}} g\left[T^{n}\left(t_{0}\right)\right]
$$

does not converge.

In other words, the $k$ th derivative of $f$ either does not exist at $t_{0}$, or if it exists, it is not equal to the sum of the derivatives of the $a^{-n} g \circ T^{n}$. Thus the $k$ th derivative of $f$ does not have regular thermodynamic limits.

Proof. Assume without loss of generality that $T$ has an unstable fixed point at $t=0$. There exists then a function $h$ analytic near $t=0$ such that $h^{-1}$ is analytic and

$$
T(t)=h\left[\lambda h^{-1}(t)\right], \quad \lambda=\partial T(0) / \partial t, \quad|\lambda|>1 .
$$

Hence, setting $g_{n}(t)=g\left[T^{n}(t)\right]$, we have

$$
g_{n}[h(t)]=G\left(\lambda^{n} t\right),
$$

with $G(t)=g[h(t)]$. Thus

$$
\partial_{t}^{k} g_{n}[h(t)]=\lambda^{n k}\left(\partial_{t}^{k} G\right)\left(\lambda^{n} t\right)
$$

Since $h(0)=0$, we see from the chain rule of differentiation that

$$
\left.\partial_{1}^{k} g_{n}(t)\right|_{t=0}=\left.\partial_{t}^{k} g_{n}\left[h\left(h^{-1}(t)\right)\right]\right|_{t=0}=\sum_{m=1}^{\infty} \lambda^{n m} a_{m k}
$$

where

$$
a_{m k}=\partial^{m} G(0) \sum_{\substack{\sum j_{p}=m \\ \sum p_{p}=k}} k ! \prod_{p=1}^{\infty}\left(\frac{\left(\partial^{p} h^{-1}\right)(0)}{p !}\right)^{j_{p}} / j_{p} !
$$

Note that $a_{m k}$ is independent of $n$. Generically, $a_{k k} \neq 0$ and hence

$$
\left.\partial_{t}^{k} g_{n}(t)\right|_{t=0}=O\left(\lambda^{n k}\right) \text {. }
$$

Thus the sum (22) will diverge for sufficiently large $k$. One can also derive (9)-(10) from this.

Corollary 4. The conclusions of lemma 3 carry over to the unstable periodic points and their pre-images.

When $T$ has no stable periodic points then we need somewhat stronger assumptions on $T$ to get interesting results.

In addition to the preceding assumptions we require $T$ to have a negative Schwarzian derivative, i.e.

$$
2 T^{\prime \prime \prime}(t) T^{\prime}(t)-3 T^{\prime \prime}(t)^{2} \leqslant 0
$$

for $t \in[0,1]$ and $T^{\prime \prime}(1 / 2)<0, T^{3}(1 / 2) \geqslant T^{2}(1 / 2)$. Again the map $T(t)=\mu t(1-t)$ and the functions of $\S 5$ satisfy these assumptions. These assumptions are very common in the study of maps of the interval (Collet and Eckmann 1980). 
Lemma 5. If $T$ has no stable periodic orbit then $f$ is 'nowhere analytic' in the following sense. There is a dense set of points in the interval $\left[T^{2}(1 / 2), T(1 / 2)\right]$ such that for each point in this set and for generic $g$ there is a $k$ for which the sum (22) diverges.

There are maps for which $k$ must grow beyond bounds, e.g. the map of Feigenbaum which is the accumulation point of periods $2^{n}$,

$$
T(x)=3.569945 \ldots x(1-x)
$$

can be shown to be of this type.

There is a situation in which one can show $k$ is bounded.

Lemma 6. If the iterate of $1 / 2$ under $T$ falls on an unstable periodic point (or fixed point) then $T$ has no stable periodic orbit, and there is a $k$ (depending only on $T$ ) for which the sum (22) diverges for generic $g$ on a dense set of points in $\left[T^{2}(1 / 2), T(1 / 2)\right]$.

Note that $T(x)=4 x(1-x)$ is such a map.

Proof of lemmas 5 and 6 . These results are easy consequences of a deep theory by Guckenheimer and Misiurewicz (see Collet and Eckmann 1980). Under our assumptions the pre-images of $1 / 2$ are dense in $\left[T^{2}(1 / 2), T(1 / 2)\right]$. We shall show that arbitrarily close to $T(1 / 2)$ there are periodic points of $T$ (in the case of lemma 6 , $T(1 / 2)$ itself is a pre-image of a periodic point). Indeed, since the pre-images of $T(1 / 2)$ are dense, we find a pre-image $x<T(1 / 2)$ of $1 / 2$ as close to $T(1 / 2)$ as we wish. Then $T^{n}(x)=T(1 / 2)$ for some $n$ but $T^{n}[T(1 / 2)]<T(1 / 2)$ since $T(1 / 2)$ is not periodic and $T(1 / 2)$ is the maximum of $T$. Thus there is an unstable periodic point between $x$ and $T(1 / 2)$. Next consider any two pre-images of $1 / 2$, say $x_{1}<x_{2}$ and assume without loss of generality that $T^{n_{1}}\left(x_{1}\right)=T(1 / 2), T^{n_{2}}\left(x_{2}\right)=T(1 / 2)$ and $n_{2}<n_{1}$ (the case of equality need not be considered since the pre-images are dense, and there is only a finite number of pre-images with fixed $n_{1}$ ). Then we apply the preceding argument to $x=T^{n_{1}-n_{2}}\left(x_{1}\right)$, yielding a pre-image of a periodic point in $\left[x_{1}, x_{2}\right]$.

So the situation is concisely described as follows: if $T$ has a stable periodic orbit, $f$ is analytic in pieces (countably many), otherwise $f$ is nowhere analytic. Unfortunately this description is only a conjecture, as it stands, since we may not exchange the sum (22) and the derivatives. Maybe the conjecture could be proved for rational maps $T$ and generic $g$.

\section{Hierarchical q-state Potts model}

It has recently been realised by McKay et al (1982) (see also Švrakić et al 1982), that certain hierarchical models with competing ferromagnetic and antiferromagnetic interactions exhibit non-monotonic renormalisation group transformations. In this section we present still another class of such frustrated models, and we illustrate for it the notions developed in the earlier sections. A more extensive discussion of such models will appear in a forthcoming publication by one of us (Erzan 1982). 
The class of models we consider are q-state Potts models (Potts 1952) with antiferromagnetic interactions. The Hamiltonian is given by

$$
-\beta H[J, \sigma]=q J \sum_{\langle i j\rangle} \delta_{\sigma_{i} \sigma_{j}},
$$

with the sum is over all nearest neighbours, $\delta_{\sigma \sigma^{\prime}}$ is the Krönecker delta, and the Potts 'spins' can take the values $\sigma=1,2, \ldots, q$. The spins are situated at the vertices of an infinite hierarchical lattice constructed via the recursive rule illustrated in figure 5 (Kaufman and Griffiths 1981).

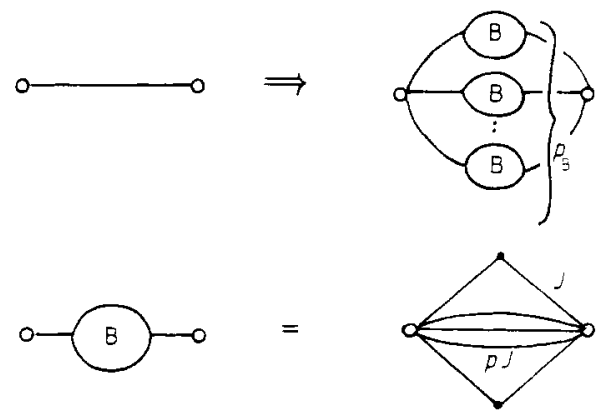

Figure 5. The recursive rule for constructing the hierarchical lattice considered in $\S 5 . p_{B}$ is the number of $B$ clusters in parallel that are substituted in place of a bond at each step. The cluster $B$ has $p$ bonds in parallel connecting the vertices denoted by open circles.

The partition function is

$$
Z=\sum_{\{\sigma\}} \exp (-\beta H[J, \sigma])
$$

The trace over all the $\sigma$ can be written as a sum of partial traces over sets of spins at sites generated by successive applications of the rule given in figure 5. Performing any such partial trace gives rise to an exact renormalisation transformation for the effective Hamiltonian. The recursion relation for the coupling constant is

where

$$
q J_{n+1}=p_{\mathrm{B}}\left[p q J_{n}+2 \log \left(\frac{1+(q-1) t_{n}^{2}}{1-t_{n}^{2}}\right)\right],
$$

$$
t_{n}=\left[1-\exp \left(-q J_{n}\right)\right] /\left[1+(q-1) \exp \left(-q J_{n}\right)\right]
$$

The renormalised Hamiltonian acquires at each step an additive constant given by

$$
g\left(t_{n}\right)=2 p_{\mathrm{B}} \log \left[\left(1+t_{n}\right) /\left(1-t_{n}\right)\right]
$$

from which the free energy per bond, as a function of the effective temperature $t$, can be calculated in the limit $n \rightarrow \infty$

$$
f\left(t_{0}\right)=\sum_{n=0}^{\infty} g\left(t_{n}\right) / a^{n}
$$

where $a=p_{\mathrm{B}}(4+p)$.

The map given by (24) and the function $g(t)$ given by (25) have the properties given in $\S 4$, up to a normalisation by $u$, for $0 \leqslant q \leqslant 2$, in the interval $[u, 0]$, where $u$ is to be found from $T(u)=0,(u \neq 0)$, for $p p_{\mathrm{B}}>1$ and $p<p^{*}(q)$ or $p_{\mathrm{B}}<\mathrm{p}_{\mathrm{B}}^{*}(q)$ where 


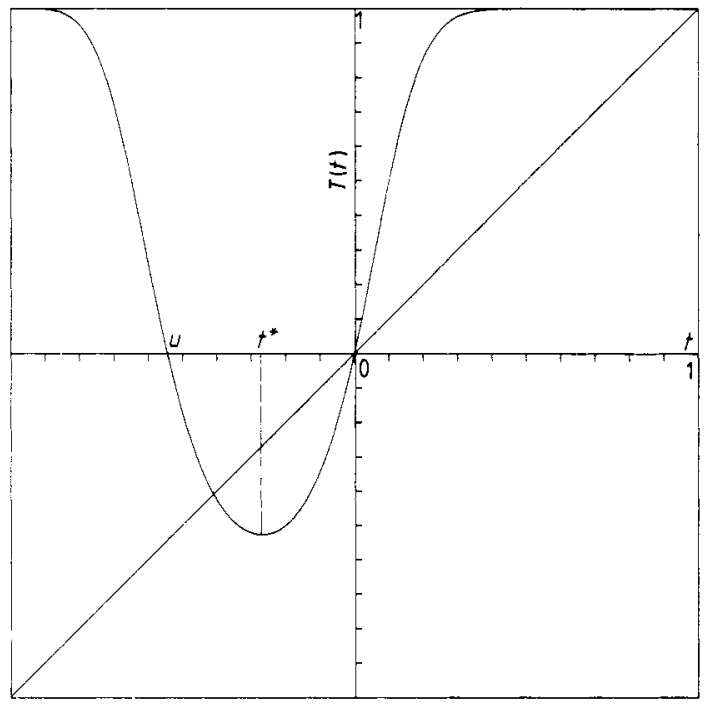

Figure 6. The map $T(t)$ induced by the renormalisation transformation on the hierarchical lattice considered in $\$ 5$ (equation (24)), for $q=2, p=1, p_{\mathrm{B}}=4.48$.

$p^{*}$, or $p_{\mathrm{B}}^{*}$, and $t^{*}$ are given by

$$
T_{p^{*}\left(p_{\mathrm{B}}^{*}\right)}^{\prime}\left(t^{*}\right)=0, \quad T_{p^{*}\left(p_{\mathrm{B}}^{*}\right)}\left(t^{*}\right)=u .
$$

For $p>p^{*}$ or $p_{\mathrm{B}}>p_{\mathrm{B}}^{*}$, the interval $[u, 0]$ is no longer mapped into itself, and all the points flow to $t=1$.

It is clear that $q$, the number of Potts states, enters these relations as a parameter, and can formally be taken to be continuous. We then find, for $0 \leqslant q<2$, that varying $p$ in the above recursion relations with $p_{\mathrm{B}}=1$ leads to period doubling and chaotic behaviour for $1<p<3$ (the range of $p$ depending on the choice of $q$ ), and for $q=2, p=1,3<p_{\mathrm{B}}<4.7$.

The singular behaviour of the fifth derivative of the free energy for the simple case of $q=2, p=1$, is illustrated in figure 7 , for $p_{\mathrm{B}}=4.48$ (period 3 ).

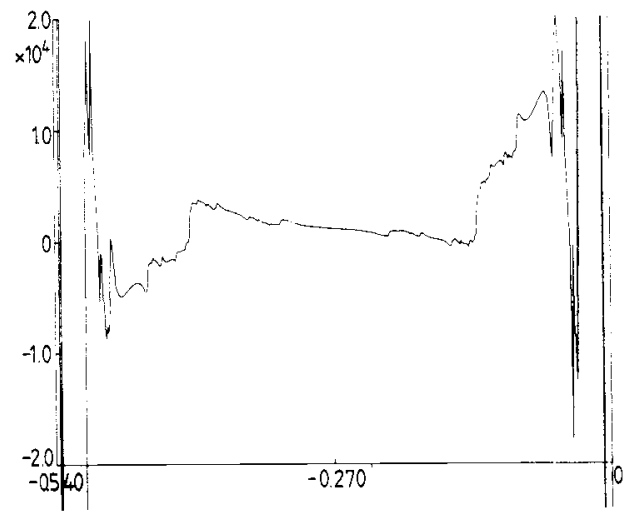

Figure 7. A combination of the first to the fifth derivatives of the free energy for the hierarchical model considered in $\S 5$, with $q=2, p=1$, for the map with stable period 3 $\left(p_{\mathrm{B}}=4.48\right)$. 
What has been calculated is the quantity

$$
\sum_{n=0}^{\infty}\left(\partial^{5} g\right)\left[T^{n}(t)\right] \prod_{i=1}^{n}\left\{T^{\prime}\left[T^{i-1}(t)\right]\right\}^{5},
$$

which is a combination of $\partial_{t}^{k} f, k \leqslant 5$, see equation (23). Although the singularities are only visible at high derivatives, the resemblance with the figures of $\S 3$ is evident.

\section{Conclusion}

We have studied the analytic properties of the free energy when the RG transformation has more complicated behaviour than only fixed points. We have shown that the main consequence is that the free energy has in general an infinite number of singularities, which can be identified with critical temperatures. Among these critical temperatures, some are accumulation points of other critical temperatures. We have not tried to identify the phases (McKay et al 1982, Švrakić et al 1982) or the order parameters because we have focused our work on the analytic properties of the function defined by (1). Our main findings are:

(i) it seems possible to construct RG maps for which the free energy is non-analytic over a range of temperatures, i.e. where every temperature is a critical temperature;

(ii) even if the RG transformation has a short attractive period, the free energy may have an infinite number of critical points.

There are several physical systems known to have whole ranges of criticality, like the line of fixed points in the two-dimensional Baxter model and the two-dimensional $X Y$ model (Baxter 1971, Kosterlitz 1974) or the Griffiths (1969) singularities in diluted random systems. We believe the phenomena described in the present paper have a different origin.

\section{References}

Baxter R J 1971 Phys. Rev. Lett. 26832

Berker A N and Ostlund S 1979 J. Phys. C: Solid State Phys. 124961

Collet P and Eckmann J-P 1980 Iterated Maps on the Interval as Dynamical Systems (Boston: Birkhäuser) Erzan A 1982 UGVA Preprint 1982/07-362

Griffiths R B 1969 Phys. Rev. Lett. 2317

Kadanoff L P 1976 Ann. Phys. 100359

Kaufman M and Griffiths R B 1981 Phys. Rev. B 24496

Kosterlitz J M 1974 J. Phys. C: Solid State Phys. 71046

McKay S, Berker A N and Kirkpatrick S 1982 Phys. Rev. Lett. 48767

May R B 1976 Nature 261459

Niemeijer Th and van Leeuwen J M J 1976 Phase Transitions and Critical Phenomena vol 6, ed C Domb and M S Green (New York: Academic)

Potts R B 1952 Proc. Camb. Phil. Soc. 48106

Šarkovskii A N 1964 Ukr. Mat. 21661

Švrakić N M, Kertész J and Selke W 1982 J. Phys. A: Math. Gen. 15 L427

Wilson K G and Kogut J 1974 Phys. Rep. 1276 